\title{
USO DE ROCAS SEDIMENTARIAS EN EL VALLE DE HUALFÍN PREHISPÁNICO
}

\section{USE OF SEDIMENTARY ROCKS IN THE PREHISPANIC HUALFIN VALLEY}

\author{
Marina Cecilia Flores
}

\begin{abstract}
1 Laboratorio de Análisis Cerámico, Facultad de Ciencias Naturales y Museo, Universidad Nacional de La Plata, calle $64 \mathrm{n}^{\circ} 3$ Laboratorio $\mathrm{n}^{\circ} 18$. La Plata; E-mail: marin481@hotmail.com
\end{abstract}

Presentado el: 12/02/2014 - Aceptado 16/10/2014

\section{Introducción}

Los materiales líticos, que por sus características intrínsecas superviven a lo largo del tiempo, constituyen una importante evidencia acerca de las actividades, movilidad y relaciones sociales de los grupos humanos del pasado. Es por ello, que desde hace varios años, se vienen desarrollando investigaciones sobre la procedencia y uso de distintos recursos líticos en el valle de Hualfín (Departamento de Belén, Catamarca).

En esta nota se presentan las rocas sedimentarias identificadas en diferentes sitios del área de estudio con evidencias de ocupación durante el Período de Desarrollos Regionales/ Inka (ca.1000-1535 AD), con la intención de abordar el origen de estos recursos y su tratamiento y uso en diferentes actividades.

\section{Los sitios considerados}

Los sitios considerados en este estudio presentan diferencias en cuento a ubicación dentro del valle, topografía, cantidad y tipos de construcciones y presencia o no de murallas. Loma de los Antiguos (Asampay) y Cerro Colorado (La Ciénaga de Abajo) corresponden a sitios fortificados situados en lomadas altas con numerosos recintos (agrupados y aislados) y murallas de defensa; Loma de Ichanga (La Ciénaga de Abajo) a asentamientos en lomadas bajas, con recintos aislados y sin muros defensivos; Campo de Carrizal (Carrizal de Asampay) a recintos y estructuras dispersos entre andenes de cultivo sobre piedemontes; y Lajas Rojas 2, Lajas Rojas 4 y Barrancas (La Ciénaga de Abajo) a recintos y estructuras dispersos, 


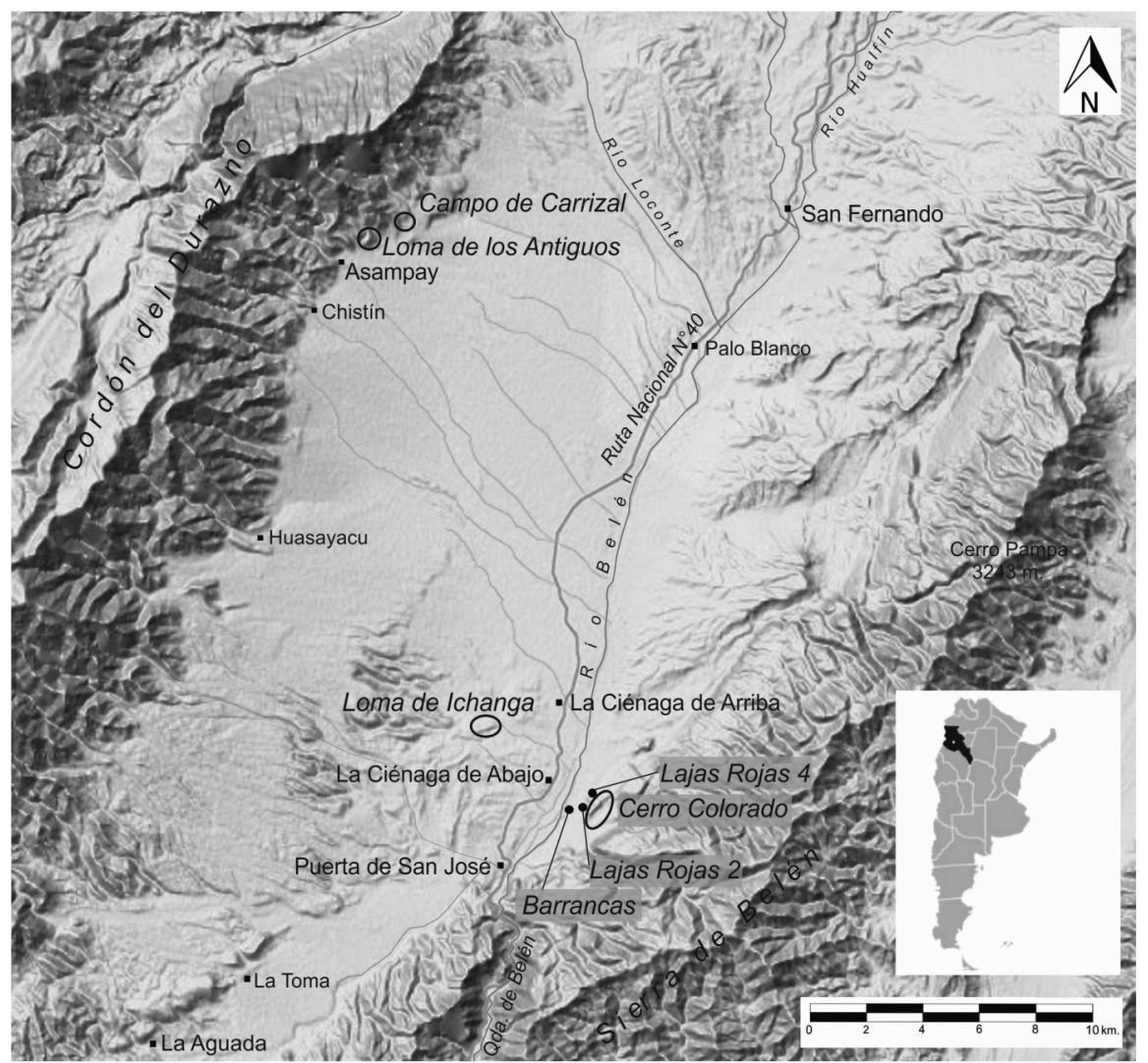

Figura 1. Los sitios considerados del valle de Hualfín (tomado y modificado de Wynveldt y Flores 2013).

al pie del Cerro Colorado y sobre la barranca del río Hualfín. A partir de la presencia de cerámica Belén Negro sobre Rojo, de las características constructivas y de los fechados radiocarbónicos, es posible sostener la ocupación de estos sitios entre los siglos XIV y XVII (Wynveldt y Flores 2013; Figura 1).

En ellos se ha identificado el uso, en diversas actividades, de rocas sedimentarias procedentes de distintas fuentes potenciales de aprovisionamiento, entre otras materias primas líticas (rocas volcánicas y minerales).

\section{Determinación y procedencia de las rocas sedimentarias}

A partir de la petrografía (Flores 2010, 2013; Flores et al. 2010, 2013), de la información geológica (González Bonorino 1950, 1972; Ruiz Huidobro 1975) y de las prospecciones realizadas por la autora en la zona de estudio se han identificado -con la colaboración de un geólogo, el Lic. Martín Morosi (Centro de Tecnología de recursos Minerales y Cerámica, CIC-CONICET)- tanto las rocas sedimentarias utilizadas por los antiguos pobladores de los sitios mencionados como sus fuentes potenciales de procedencia. Se trata de areniscas -cuarzoarenita (CZA), feldarenita (FA), litoarenita (LA), sublitoarenita (SLA), arenita deleznable (AD) y arenita indeterminada (AI)- y limolitas -L1, L2, L3, L4 y L5-. Son recursos locales, obtenidos a diferentes distancias de los sitios, probablemente de forma directa (Meltzer 1989). La mayor parte se presentan bajo la forma de cantos rodados, de diferentes dimensiones y 
formas, en los cauces de ríos, antiguas terrazas fluviales y barrancas -fuentes secundarias-; excepto la SLA, AD y L4, cuyos afloramientos -fuentes primarias- se encuentran sobre la margen oriental del río Hualfín.

\section{Tratamiento y uso de las rocas sedimentarias en distintas actividades}

Las rocas sedimentarias estudiadas se encuentran vinculadas con la realización de diferentes actividades, tales como la talla, la molienda y la construcción (Tabla 1). A continuación se aborda el tratamiento y uso de estos recursos para cada una de las actividades mencionadas. Para el análisis de los artefactos líticos tallados se siguió a Aschero (1975 y Rev. 1983) y para el de los artefactos líticos de molienda a Babot (2004).

Talla

Las rocas sedimentarias utilizadas en esta actividad conforman entre el 17\% y el 79\% del conjunto total de materias primas de los sitios contemplados y entre el $66 \%$ y el $100 \%$, si se tienen en cuenta sólo las locales (Tabla 1).

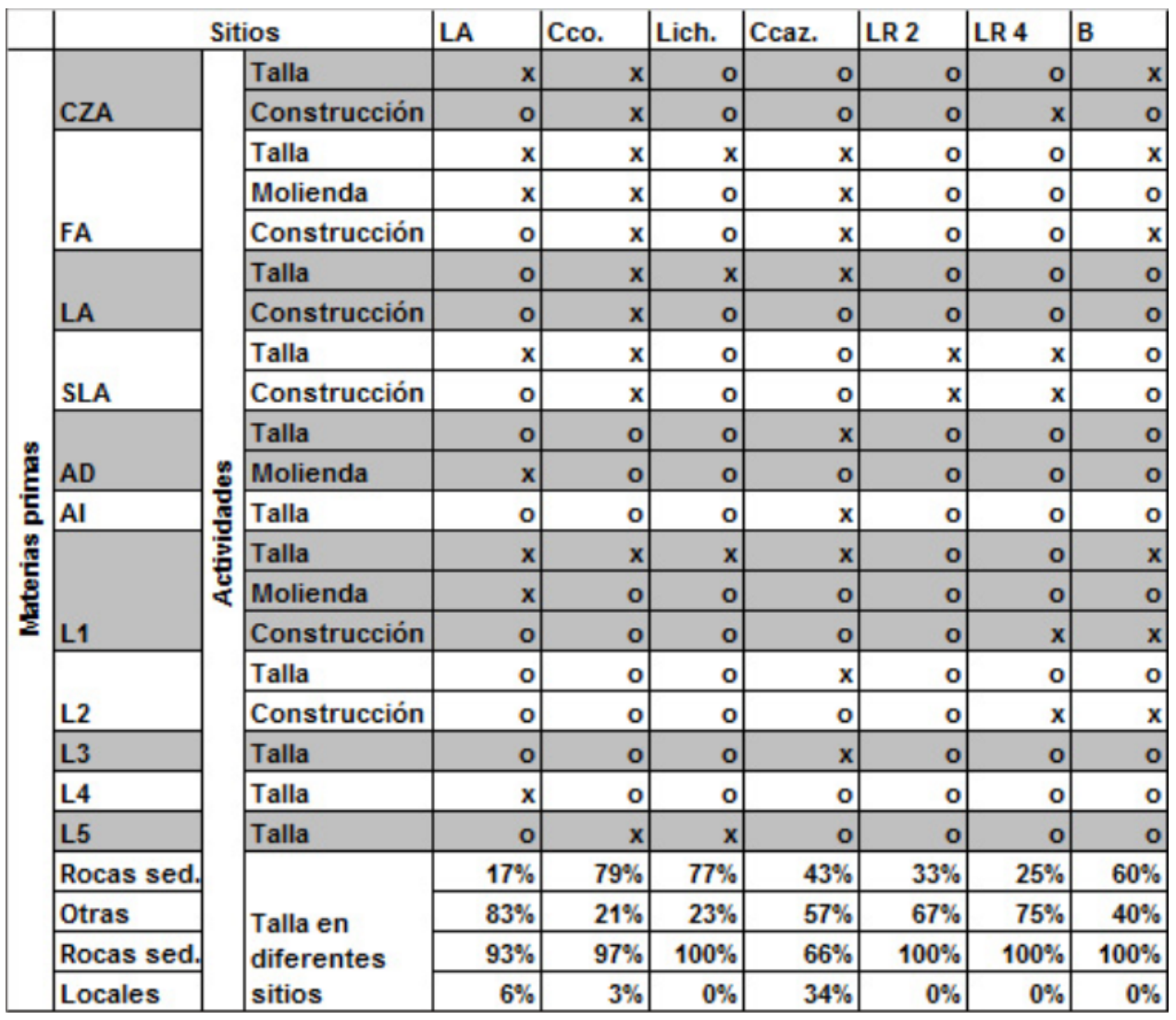

Tabla 1. Materias primas y actividades desarrolladas por sitio y proporción de rocas sedimentarias utilizadas en la talla en diferentes sitios. Referencias: -sitios- LA (Loma de los Antiguos), Cco. (Cerro Colorado), LIch. (Loma de Ichanga), Ccaz.(Campo de Carrizal), LR2 (Lajas Rojas 2), LR4 (Lajas Rojas 4) y B (Barrancas);-materias primas- CZA (cuarzoarenita), FA (feldarenita), LA (litoarenita), SLA (sublitoarenita), AD (arenita deleznable), AI (arenita indeterminada), L1 (limolita 1), L2 (limolita 2), L3 (limolita 3), L4 (limolita 4) y L5 (limolita 5). 


\begin{tabular}{|c|c|c|c|c|c|c|c|c|}
\hline \multirow{2}{*}{$\begin{array}{c}\text { Materias } \\
\text { primas }\end{array}$} & \multicolumn{7}{|c|}{ Clases tipológicas } & \multirow{2}{*}{ Total } \\
\hline & AF & ANFCRC & DT & N & AND & HG & $\mathbf{P}$ & \\
\hline CZA & 1 & 1 & 12 & 0 & 0 & 0 & 0 & 14 \\
\hline FA & 6 & 2 & 117 & 4 & 1 & 1 & 1 & 132 \\
\hline LA & 0 & 0 & 5 & 1 & 0 & 0 & 0 & 6 \\
\hline SLA & 3 & 0 & 46 & 0 & 0 & 0 & 0 & 49 \\
\hline Al & 0 & 0 & 1 & 0 & 0 & 0 & 0 & 1 \\
\hline$A D$ & 0 & 0 & 1 & 0 & 0 & 0 & 0 & 1 \\
\hline L1 & 2 & 0 & 34 & 1 & 0 & 0 & 0 & 37 \\
\hline L2 & 0 & 0 & 6 & 0 & 0 & 0 & 0 & 6 \\
\hline L3 & 0 & 0 & 17 & 0 & 0 & 0 & 0 & 17 \\
\hline L4 & 1 & 1 & 0 & 0 & 0 & 0 & 0 & 2 \\
\hline L5 & 1 & 1 & 4 & 1 & 0 & 0 & 0 & 7 \\
\hline Total & 14 & 5 & 243 & 7 & 1 & 1 & 1 & 272 \\
\hline
\end{tabular}

Tabla 2. Clases tipológicas y materias primas. Referencias: -materias primas- CZA (cuarzoarenita), FA (feldarenita), LA (litoarenita), SLA (sublitoarenita), AI (arenita indeterminada), AD (arenita deleznable), L1 (limolita 1), L2 (limolita 2), L3 (limolita 3), L4 (limolita 4) y L5 (limolita 5); -clases tipológicas- AF (artefacto formatizado), ANFCRC (artefacto no formatizado con rastros complementarios), DT (desechos de talla), N (núcleos), AND (artefacto no diferenciado), HG (hemiguijarro) y $\mathrm{P}$ (percutor).

\begin{tabular}{|c|c|c|c|c|c|c|c|}
\hline \multirow{2}{*}{$\begin{array}{c}\text { Materias } \\
\text { primas }\end{array}$} & \multirow{2}{*}{ Grupos tipológicos } & \multirow{2}{*}{ Formas bases } & \multicolumn{5}{|c|}{ Sitios } \\
\hline & & & LA & Cco. & Lich. & Ccaz. & B \\
\hline CZA & $\begin{array}{l}\text { Raedera+Artefacto de } \\
\text { formatización sumaria }\end{array}$ & no diferenciada & 1 & 0 & 0 & 0 & 0 \\
\hline \multirow{6}{*}{ FA } & $\begin{array}{l}\text { Artefacto de formatización } \\
\text { sumaria (2)+Raspador }\end{array}$ & lasca & 1 & 0 & 0 & 0 & 0 \\
\hline & Raspador & lasca & $\mathbf{0}$ & 0 & 0 & 1 & 0 \\
\hline & $\begin{array}{l}\text { Denticulado+RBO+Artefacto de } \\
\text { formatización sumaria }\end{array}$ & lasca & 0 & 0 & 0 & 1 & 0 \\
\hline & Denticulado & hemiguijarro & $\mathbf{0}$ & 0 & 0 & 1 & 0 \\
\hline & $\begin{array}{c}\text { Artefacto de formatización } \\
\text { sumaria+Raspador+Artefacto de } \\
\text { formatización sumaria (3) }\end{array}$ & lasca & 0 & 0 & 1 & 0 & 0 \\
\hline & Cepillo & $\begin{array}{c}\text { guijarro/artefacto } \\
\text { de molienda }\end{array}$ & $\mathbf{0}$ & 0 & 0 & 2 & 0 \\
\hline \multirow{3}{*}{ SLA } & $\begin{array}{l}\text { Denticulado+Artefacto de } \\
\text { formatización sumaria }\end{array}$ & no diferenciada & 0 & 1 & 0 & 0 & 0 \\
\hline & Denticulado & clasto natural & 0 & 1 & 0 & 0 & 0 \\
\hline & Raspador & lasca & 0 & 1 & 0 & 0 & 0 \\
\hline \multirow{2}{*}{ L1 } & Raedera & lasca & 1 & 0 & 0 & 0 & 0 \\
\hline & No diferenciado & lasca & $\mathbf{0}$ & 0 & 0 & 1 & 1 \\
\hline L4 & $\begin{array}{c}\text { Punta burilante } \\
\text { angular }+ \text { RBO+Raspador+Muesca }\end{array}$ & lasca & 1 & 0 & 0 & 0 & 0 \\
\hline L5 & Muesca & lasca & 0 & 0 & 1 & 0 & 0 \\
\hline
\end{tabular}

Tabla 3. Grupos tipológicos y formas base de artefactos formatizados por materias primas y sitio. Referencias: materias primas- CZA (cuarzoarenitas), FA (feldarenita), SLA (sublitoarenita), L1 (limolita 1), L4 (limolita 4) y L5 (limolita 5); -grupos tipológicos- RBO (artefacto retocado en bisel asimétrico oblicuo) 
A partir del análisis conjunto de todos los artefactos tallados recuperados se observa, en orden decreciente, la presencia de las siguientes rocas: FA, SLA, L1, L3, CZA, L5, LA, L2, L4, AD y AI; las cuales se encuentran representadas principalmente, por desechos de talla (Tabla 2). Sólo en seis variedades de rocas sedimentarias se reconocieron artefactos formatizados -simples y compuestos-, entre los cuales los filos más recurrentes corresponden a artefacto de formatización sumaria, raspador y denticulado (Tabla 3). Los mismos están presentes en distintas materias primas. Las formas bases utilizadas son varias, siendo preferentes las lascas. En cuanto a los desechos, la mayoría se encuentran fragmentados, las formas de lascas más frecuentes corresponden a angulares, seguidas por indiferenciadas (Tabla 4); los talones corticales son los más numerosos seguidos por lisos y astillados (Tabla 4). Por último, los núcleos (tabla 5) son escasos y la morfología discoidal irregular es la más representada, además de constituir la única registrada en varias materias primas. Las formas bases son guijarros, excepto un caso en FA, donde el soporte corresponde a un artefacto de molienda.

\section{Molienda}

En base a los hallazgos en superficie y excavación de tres sitios (Tabla 1) se aprecia que las rocas sedimentarias representan aproximadamente el 33\% de las materias primas vinculadas con esta actividad, siendo las restantes rocas ígneas e indeterminadas. De mayor a menor frecuencia corresponden a: FA, AD y L1. Se trata principalmente de partes activas de artefactos de molienda -siete manos de molino (FA, AD y L1), dos manos de mortero (FA), una mano de molino/mortero (FA)- y dos morteros (FA). Las manos se encuentran enteras y fragmentadas en la misma proporción. La mayoría de las manos de molino presentan dos caras activas opuestas, excepto un caso en L1. Sus formas bases corresponden a guijarros de diferentes formas y dimensiones, que no presentan modificaciones; excepto unos pocos que habrían sido acondicionados en cuanto a su morfología final o para una mejor prensión del implemento. Por otra parte, los morteros son móviles (FA), uno presenta forma base guijarro y tiene abierta una oquedad central, mientras el segundo corresponde a un bloque plano sobre la superficie del cual se realizaron varias oquedades de diámetro pequeño.

\begin{tabular}{|c|c|c|c|c|c|c|c|c|c|c|c|c|c|c|c|c|c|}
\hline \multirow{2}{*}{$\begin{array}{l}\text { Materias } \\
\text { primas }\end{array}$} & \multicolumn{8}{|c|}{ Formas de lascas } & \multirow[b]{2}{*}{ Total } & \multicolumn{7}{|c|}{ Formas de talones } & \multirow[b]{2}{*}{ Total } \\
\hline & LP & LS & LCDN & LAS & LA & LPL & LB & $\mathbf{L I}$ & & c & $\mathrm{Li}$ & D & $\mathrm{F}$ & $\mathrm{Fi}$ & A & ND & \\
\hline CZA & 2 & 1 & 2 & 0 & 5 & 0 & 0 & 1 & 11 & 5 & 4 & 0 & 0 & 0 & 1 & 1 & 11 \\
\hline FA & 3 & 0 & 1 & 8 & 47 & 2 & 0 & 51 & 112 & 32 & 26 & 1 & 1 & 2 & 6 & 0 & 68 \\
\hline LA & 1 & 1 & 1 & 0 & 2 & 0 & 0 & 0 & 5 & 4 & 0 & 0 & 0 & 0 & 1 & 0 & 5 \\
\hline SLA & 2 & 2 & 0 & 4 & 31 & 0 & 0 & 7 & 46 & 17 & 14 & 1 & 1 & 0 & 0 & 0 & 33 \\
\hline Al & 0 & 0 & 0 & 0 & 0 & 0 & 0 & 1 & 1 & 0 & 0 & 0 & 0 & 0 & 1 & 0 & 1 \\
\hline$A D$ & 0 & 0 & 0 & 0 & 0 & 0 & 0 & 1 & 1 & 0 & 1 & 0 & 0 & 0 & 0 & 0 & 1 \\
\hline L1 & 0 & 1 & 2 & 1 & 18 & 0 & 0 & 11 & 33 & 4 & 9 & 0 & 0 & 2 & 5 & 1 & 21 \\
\hline L2 & 0 & 0 & 0 & 0 & 2 & 0 & 1 & 3 & 6 & 2 & 0 & 0 & 0 & 0 & 1 & 0 & 3 \\
\hline L3 & 0 & 0 & 1 & 0 & 10 & 0 & 0 & 6 & 17 & 4 & 1 & 1 & 0 & 1 & 2 & 0 & 9 \\
\hline L5 & 0 & 2 & 0 & 0 & 2 & 0 & 0 & 1 & 5 & 1 & 2 & 0 & 0 & 0 & 0 & 0 & 3 \\
\hline Total & 8 & 7 & 7 & 13 & 117 & 2 & 1 & 82 & 237 & 69 & 57 & 3 & 2 & 5 & 17 & 2 & 155 \\
\hline
\end{tabular}

Tabla 4. Formas de lascas y talones por materias primas. Referencias: -materias primas- CZA (cuarzoarenita), FA (feldarenita), LA (litoarenita), SLA (sublitoarenita), AI (arenita indeterminada), AD (arenita deleznable), L1 (limolita 1), L2 (limolita 2), L3 (limolita 3) y L5 (limolita 5); -formas de lascas- LP (lasca primaria), LS (lasca secundaria), LCDN (lasca con dorso natural), LAS (lasca de arista simple), LA (lasca angular), LPL (lasca plana), LB (lasca bipolar) y LI (lasca indiferenciada); -formas de talones- C (cortical), Li (liso), D (diedro), F (facetado), Fi (filiforme), A (astillado) y ND (no diferenciado). 


\begin{tabular}{|c|c|c|c|c|c|}
\hline \multirow{2}{*}{ Materias primas } & \multicolumn{4}{|c|}{ Designación morfológica } & \multirow{2}{*}{ Total } \\
\cline { 2 - 6 } & Discoidal irregular & Poliédrico & Poliédrico incompleto & Bifacial irregular & \\
\hline FA & 2 & 1 & 1 & 0 & 4 \\
\hline LA & 1 & 0 & 0 & 0 & 1 \\
\hline L1 & 0 & 0 & 0 & 1 & 1 \\
\hline L5 & 1 & 0 & 0 & 0 & 1 \\
\hline
\end{tabular}

Tabla 5. Designación morfológica y materias primas. Referencias: -materias primas- FA (feldarenita), LA (litoarenita), L1 (limolita 1) y L5 (limolita 5).

\section{Construcción}

Las construcciones observadas corresponden principalmente a recintos, murallas y cistas. Entre las rocas sedimentarias registradas en la construcción (Tabla 1) se observa que aquellas que se presentan bajo la forma de cantos rodados de recintos -CZA, FA, LA y L1han sido utilizadas sin modificación alguna y como materiales constructivos secundarios o minoritarios acompañando otros tipos de rocas y minerales, excepto en el sitio Barrancas. En éste último, los cantos rodados enteros y fragmentados, constituyen el único tipo de material lítico constructivo. Por otro lado, la SLA ha sido fraccionada, en las fuentes primarias, en bloques cuadrangulares de diferentes dimensiones para utilizarse, de manera preferencial o exclusiva, en las construcciones -recintos, murallas o cistas- en varios sitios (Tabla 1).

\section{Discusión y conclusiones}

En los sitios considerados aquí se han utilizado rocas sedimentarias -areniscas y limolitas- en las actividades de talla, molienda y construcción. Estos recursos son locales y habrían sido obtenidos de forma directa a partir de fuentes potenciales de aprovisionamiento primarias y secundarias.

La mayor variedad de rocas se encuentra vinculada con la talla, siendo menor la identificada en las actividades de molienda y construcción. Solo la feldarenita y la limolita 1 están relacionadas con las tres actividades. La presencia y cantidad de los recursos en los diferentes sitios estaría supeditada, principalmente, a la cercanía de las fuentes potenciales de aprovisionamiento y a su abundancia. Asimismo, pudieron existir otras cuestiones -morfológicas, estéticas, simbólicas, de poder, perceptivas, paisajísticas y temporales que intervinieron en la elección. La información obtenida no permite interpretar que la diferencia entre los sitios, en cuanto a su función o complejidad haya actuado a favor o en contra del acceso y uso de estos recursos.

Para la talla, se observa que las rocas procedentes de fuentes secundarias habrían sido introducidas a los sitios sin transformación alguna; en cambio las obtenidas en afloramientos primarios, habrían sido modificadas al momento de su extracción. A partir del análisis realizado sobre los artefactos tallados es posible sostener: el desarrollo de talla directa con percutor duro -sólo la limolita 1 presenta, además, evidencias de uso de percutor blando-, el desbaste a partir de múltiples planos de percusión y la ausencia de preparación y mantenimiento de núcleos. En general, la talla habría estado dirigida a la obtención de lascas para la formatización de filos -entre los más recurrentes: formatización sumaria, raspadores y denticulados- o el uso potencial de filos naturales. La formatización sumaria observada 
en algunos artefactos se encuentra acompañando a otros filos y podría vincularse con un acondicionamiento para la prensión manual del ítem o con la formatización destacada de otros filos. Las materias primas que presentan mayor diversidad de filos son la feldarenita y la limolita 4. La identificación de los mismos grupos tipológicos en diferentes materias primas, desestima la posibilidad de que exista una relación filo-roca específica, aunque quizás sí granulométrica. Esto último podría estar relacionado con la presencia de denticulados y cepillos sólo en areniscas y muescas, únicamente, en limolitas. Por otra parte, es interesante señalar el uso de antiguos artefactos de molienda en feldarenita como formas base para núcleos y artefactos formatizados, lo cual permite plantear el aprovisionamiento de este recurso en fuentes terciarias -sitio- (Church 1994) y la práctica de reciclaje (Schiffer 1987). El hecho de que además, la feldarenita haya sido empleada como percutor, evidencia su amplio uso.

Para la molienda, los guijarros de rocas sedimentarias parecen haber sido los más idóneos como partes activas. Los perfiles de las manos, en general, permiten interpretar como habrían sido usadas y mantenidas (Babot 2004). En este caso se ha registrado mayormente el empleo de molinos con caras activas y dobles, que por sus perfiles y superficies de uso homogéneos permiten plantear un manejo del desgaste por parte de los operadores. Este control habría posibilitado el prolongamiento de la vida útil de un artefacto muy confortable para su uso o manufacturado en un material escaso. Por otra parte, las variables morfológicas de los morteros, como su tamaño y cantidad de oquedades implican la posibilidad de uso por uno o varios operadores en forma simultánea. No se poseen evidencias para plantear una asociación entre grupos tipológicos y tipos de rocas sedimentarias.

La distribución de los artefactos líticos en los sitios considerados (Flores 2013), de los cuales los aquí estudiados son solo una parte, permiten proponer que las actividades de talla y molienda se habrían llevado a cabo tanto dentro como fuera de los recintos y que habrían estado orientadas a cubrir las necesidades cotidianas.

En cuanto a las construcciones, podemos señalar el uso de los recursos líticos más inmediatos a cada uno de los sitios. El empleo de fuentes primarias o secundarias habría involucrado cadenas operativas de diferente complejidad, siendo más largas o con más etapas, en el caso de las primeras. La actividad constructiva habría sido una práctica grupal, por lo menos a nivel del grupo doméstico, teniendo en cuenta las dimensiones y pesos de las rocas utilizadas, así como su cantidad, entre otras cuestiones.

El empleo de los mismos tipos de rocas para las mismas actividades en diferentes sitios de los considerados aquí y el tratamiento general observado para la explotación de estos recursos permiten plantear un "saber" compartido por los pobladores del valle para estos momentos.

A partir de este trabajo se ha logrado avanzar en el conocimiento de los recursos líticos locales de origen sedimentario vinculados con diferentes prácticas sociales desarrolladas por los antiguos pobladores del valle de Hualfín.

Agradecimientos. A la Universidad Nacional de La Plata, a la Agencia Nacional de Promoción Científica y Tecnológica, al CONICET y al CETMIC., por proveer la infraestructura y medios para la realización del trabajo. Al Lic. Martín Morosi. 


\section{Bibliografía citada}

Aschero, C.

1975 Ensayo para una clasificación morfológica de artefactos líticos aplicada a estudios tipológicos comparativos. Informe presentado al CONICET.

1983 Ensayo para una clasificación morfológica de artefactos líticos. Apéndice A y B. Cátedra de Ergología y Tecnología. Copias disponibles en Facultad de Filosofía y Letras, Universidad de Buenos Aires.

Babot, M.

2004 Tecnología y utilización de artefactos de molienda en el noroeste prehispánico. Tesis Doctoral inédita. Facultad de Ciencias Naturales e I.M.L. Universidad Nacional de Tucumán.

Church, T.

1994 Terms in lithic resource studies. Lithic resource studies: a source for archaeologist. Lithic Technology 3:9-25.

Flores, $\mathrm{M}$.

2010 Actividades vinculadas a la tecnología lítica en la localidad de La Ciénaga durante el Período de Desarrollos Regionales/Inka. Aldeas protegidas, conflicto y abandono: Investigaciones arqueológicas en La Ciénaga (Catamarca, Argentina) (ed. por B. Balesta y N. Zagorodny), pp. 123-159. Al Margen, La Plata.

2013 Aprovisionamiento y manejo de materias primas líticas durante el Período de Desarrollos Regionales/Inka en el valle de Hualfin (Depto. de Belén, Catamarca). Tesis Doctoral Inédita para optar por el grado de Doctor en Ciencias Naturales y Museo, Universidad Nacional de La Plata.

Flores, M., M. Morosi y F. Wynveldt

2010 Determinación de materias primas líticas de la Loma de los Antiguos de Azampay (Dto. de Belén, Catamarca, Argentina). Revista Werken 13: 261-273.-

Flores, M., M. Morosi y N. Zagorodny

2013 Análisis petrográfico de artefactos líticos tallados del sitio Campo de Carrizal (Depto. de Belén, Catamarca) correspondientes al Período de Desarrollos Regionales/Inka. Resumen del V Congreso Nacional de Arqueometría y Primer Encuentro de Tecnologías Históricas, Rosario.

González Bonorino, F.

1950 Geología y Petrografía de las Hojas 12d (Capillitas) y 13d (Andalgalá). Ministerio de Industria y Comercio de la Nación, Dirección General de Minería. Boletín $\mathrm{N}^{\mathrm{o}} 70$. Buenos Aires.

1972 Descripción Geológica de la Hoja 13c, Fiambalá, Provincia de Catamarca. Ministerio de Industria y Minería. Subsecretaría de Minería. Dirección Nacional de Geología y Minería. Boletín $\mathrm{N}^{\circ}$ 127. Buenos Aires.

Meltzer, D.

1989 Was stone excahanged among eastern North American paleoindians? Eastern paleoindians lithic resources use, (ed. por C. J. Ellis y J. Lathrop), pp. 11-39. Westview Press, Boulder. 
Ruiz Huidobro, O.

1975 Descripción geológica de la Hoja 12 c, Laguna Helada, Provincia de Catamarca. Ministerio de Economía, Secretaria de Estado de Recursos Naturales y Ambiente Humano, Subsecretaría de Minería, Servicio Geológico Nacional. Boletín No 146. Buenos Aires.

Schiffer, M.

1987 Formation Processes of the Archaeological Record. University of Utah Press, Salt Lake City.

Wynveldt, F. y M. Flores

2013 La obsidiana en el paisaje Tardío del valle de Hualfín (Depto. de Belén, Prov. de Catamarca). Resumen del XVIII Congreso Nacional de Arqueología Argentina, La Rioja. 